\title{
The hottest horizontal-branch stars in $\omega$ Centauri. Late hot flasher vs. helium enrichment ${ }^{\star}$
}

\author{
S. Moehler ${ }^{1}$, S. Dreizler ${ }^{2}$, T. Lanz ${ }^{3}$, G. Bono ${ }^{4}$, A. V. Sweigart ${ }^{5}$, A. Calamida ${ }^{4}$, M. Monelli $^{6}$, and M. Nonino ${ }^{7}$ \\ ${ }^{1}$ European Southern Observatory, Karl-Schwarzschild-Str. 2, 85748 Garching, Germany \\ e-mail: smoehler@eso.org \\ ${ }^{2}$ Georg-August-Universität, Institut für Astrophysik, Friedrich-Hund-Platz 1, 37077 Göttingen, Germany \\ e-mail: dreizler@astro.physik.uni-goettingen.de \\ 3 Department of Astronomy, University of Maryland, College Park, MD 20742-2421, USA \\ e-mail: lanz@astro.umd.edu \\ 4 INAF - Rome Astronomical Observatory, via Frascati 33, 00040 Monte Porzio Catone, Italy \\ e-mail: [bono; calamida] @mporzio.astro.it \\ 5 NASA Goddard Space Flight Center, Code 667, Greenbelt, MD 20771, USA \\ e-mail: Allen.V.Sweigart@nasa.gov \\ ${ }^{6}$ IAC - Instituto de Astrofisica de Canarias, Calle via Lactea, 38200 La Laguna, Tenerife, Spain \\ e-mail: monelli@iac.es \\ 7 INAF - Trieste Astronomical Observatory, via G.B. Tiepolo 11, 40131 Trieste, Italy \\ e-mail: nonino@ts.astro.it
}

Received 20 August 2007 / Accepted 18 September 2007

\begin{abstract}
Context. UV observations of some massive globular clusters have revealed a significant population of very hot stars below the hot end of the horizontal branch (HB), the so-called blue hook stars. This feature might be explained either as a result of the late hot flasher scenario where stars experience the helium flash while on the white dwarf cooling curve or by the progeny of the helium-enriched subpopulation recently postulated to exist in some clusters. Previous spectroscopic analyses of blue hook stars in $\omega$ Cen and NGC 2808 support the late hot flasher scenario, but the stars contain much less helium than expected and the predicted $\mathrm{C}$, $\mathrm{N}$ enrichment could not be verified.

Aims. We compare observed effective temperatures, surface gravities, and abundances of $\mathrm{He}, \mathrm{C}$, and $\mathrm{N}$ of blue hook and canonical extreme horizontal branch (EHB) star candidates to the predictions of the two scenarios.

Methods. Moderately high resolution spectra of stars at the hot end of the blue HB in the globular cluster $\omega$ Cen were analysed for atmospheric parameters and abundances using LTE and non-LTE model atmospheres.

Results. In the temperature range $30000 \mathrm{~K}$ to $50000 \mathrm{~K}$ we find that $35 \%$ of our stars are helium-poor $\left(\log \frac{n_{\mathrm{He}}}{n_{\mathrm{H}}}<-2\right), 51 \%$ have solar helium abundance within a factor of $3\left(-1.5 \lessgtr \log \frac{n_{\mathrm{He}}}{n_{\mathrm{H}}} \lesssim-0.5\right)$ and $14 \%$ are helium-rich $\left(\log \frac{n_{\mathrm{He}}}{n_{\mathrm{H}}}>-0.4\right)$. We also find carbon enrichment strongly correlated with helium enrichment, with a maximum carbon enrichment of $3 \%$ by mass.

Conclusions. At least $14 \%$ of the hottest HB stars in $\omega$ Cen show helium abundances well above the highest predictions from the helium enrichment scenario ( $Y=0.42$ corresponding to $\left.\log \frac{n_{\mathrm{He}}}{n_{\mathrm{H}}} \approx-0.74\right)$. In addition, the most helium-rich stars show strong carbon enrichment, as predicted by the late hot flasher scenario. We conclude that the helium-rich HB stars in $\omega$ Cen cannot be explained solely by the helium-enrichment scenario invoked to explain the blue main sequence.
\end{abstract}

Key words. stars: horizontal-branch - stars: evolution - techniques: spectroscopic - Galaxy: globular clusters: individual: $\omega$ Centauri

\section{Introduction}

UV-visual colour-magnitude diagrams of the two very massive globular clusters, $\omega$ Cen and NGC 2808, show a rather puzzling "hook-like" feature at the hot end of their extended horizontal branches with stars lying below the canonical horizontal branch (Whitney et al. 1998; D'Cruz et al. 2000; Brown et al. 2001). These stars cannot be explained within the framework of canonical stellar evolution. Brown et al. (2001) have proposed a "flash-mixing" scenario to explain the blue hook stars. According to this scenario, stars that lose an unusually large

* Based on observations with the ESO Very Large Telescope at Paranal Observatory, Chile (proposal IDs 075.D-0280(A) and 077.D-0021(A)) amount of mass will leave the red giant branch (RGB) before the helium flash and will move quickly to the (helium-core) white dwarf cooling curve before igniting helium (Castellani \& Castellani 1993; D’Cruz et al. 1996; Brown et al. 2001). However, the evolution of these "late hot helium flashers" differs dramatically from the evolution of stars that undergo the helium flash on the RGB. Ordinarily, when a star flashes at the tip of the RGB or shortly thereafter, the large entropy barrier of its strong hydrogen-burning shell prevents the products of helium burning from being mixed to the surface. Such canonical stars will evolve to the zero-age horizontal branch (ZAHB) without any change in their hydrogen-rich envelope composition. In contrast, stars that ignite helium on the white dwarf cooling curve, where the hydrogen-burning shell is much weaker, will undergo 
extensive mixing between the helium- and carbon-rich core and the hydrogen envelope (Sweigart 1997; Brown et al. 2001; Cassisi et al. 2003). Depending on where the helium flash occurs along the white dwarf cooling curve, the envelope hydrogen will be mixed either deeply into the core ("deep mixing") or only with a convective shell in the outer part of the core ("shallow mixing"). In the case of deep mixing, virtually all of the envelope hydrogen is burned while in shallow mixing some of the envelope hydrogen remains after the mixing phase (Lanz et al. 2004). One of the most robust predictions of the flash-mixing scenario is an increase in the surface abundance of carbon to 3-5\% (deep mixing) or $1 \%$ (shallow mixing) by mass. This increase is set by the carbon production during the helium flash and is nearly independent of the stellar parameters. Nitrogen may also be enhanced due to the burning of hydrogen on triple- $\alpha$ carbon during the flash-mixing phase. For both deep and shallow mixing, the blue hook stars should be helium-rich compared to the canonical EHB stars.

Alternatively, the recently observed split among the main sequence stars of $\omega$ Cen and NGC 2808 (Bedin et al. 2004; Piotto et al. 2005, 2007) has been attributed to a sub-population of stars with helium abundances as large as $Y \approx 0.4$ (Norris 2004; D'Antona et al. 2005; D'Antona \& Ventura 2007; see Newsham \& Terndrup 2007 for cautionary remarks). Lee et al. (2005) have suggested that the blue hook stars are the progeny of these proposed helium-rich main sequence stars. If the blue hook stars were to be explained by the helium-enrichment scenario, their helium abundance should not exceed $Y \approx 0.4$ and carbon should not be enriched at all. Spectroscopic observations of the blue (and supposedly helium-rich) main sequence stars in $\omega$ Cen yield a carbon abundance of $[\mathrm{C} / \mathrm{M}]=0.0$ (Piotto et al. 2005). This carbon abundance will decrease further as the stars ascend the red giant branch, due to the extra-mixing process that occurs in metal-poor red giants (Gratton et al. 2000; Kraft 1994). Origlia et al. (2003) have confirmed that the RGB stars in $\omega$ Cen have the low ${ }^{12} \mathrm{C} /{ }^{13} \mathrm{C}$ ratios $(\approx 4)$ and low average carbon abundances $([\mathrm{C} / \mathrm{Fe}]=-0.2)$ expected from this extra mixing. Thus the helium-enrichment scenario predicts a carbon abundance by mass in the blue hook stars of less than $0.1 \%$, i.e., at least a factor of 10 smaller than the carbon abundance predicted by the flash-mixing scenario.

Previous spectra of the blue hook stars in $\omega$ Cen (Moehler et al. 2002) and NGC 2808 (Moehler et al. 2004) showed that these stars are indeed both hotter and more helium-rich than the canonical EHB stars. However, the blue hook stars still show considerable amounts of hydrogen. Unfortunately, due to limited resolution and signal-to-noise ratios $(\mathrm{S} / \mathrm{N})$ we could not derive good abundances for $\mathrm{C}$ and $\mathrm{N}$. Instead we could only state that the most helium-rich stars appear to show some evidence for $\mathrm{C} / \mathrm{N}$ enrichment. Therefore we started a project to obtain higher resolution spectra of EHB and blue hook stars in $\omega$ Cen.

\section{Observations, data reduction, and analysis}

We selected stars along the blue HB in $\omega$ Cen from the multiband $(U, B, V, I)$ photometry of Castellani et al. (2007). These data were collected with the mosaic CCD camera Wide Field Imager available at the $2.2 \mathrm{~m} \mathrm{ESO/MPI} \mathrm{telescope.} \mathrm{The} \mathrm{field} \mathrm{of}$ view covered by the entire mosaic is $42^{\prime} \times 48^{\prime}$ across the center of the cluster. These data together with multiband data from the Advanced Camera for Surveys on board the Hubble Space Telescope provided the largest sample of HB stars $(\approx 3200)$ ever collected in a globular cluster. Among them we concentrated on the stars at the faint end of the $\mathrm{HB}$, which are the most likely "blue hook" candidates as shown by Moehler et al. (2002, 2004). In order to avoid crowding problems, we only selected isolated EHB stars. The astrometry was performed using the UCAC2 catalog (Zacharias et al. 2004), which does not cover the central crowded regions. However, thanks to the large field covered by current dataset the astrometric solution is based on $\approx 3000$ objects with an rms error of 0. '06.

The spectroscopic data were obtained in 2005 (4 observations) and in 2006 (5 observations) in Service Mode using the MEDUSA mode of the multi-object fibre spectrograph FLAMES+GIRAFFE on the UT2 Telescope of the VLT. We used the low spectroscopic resolution mode with the spectral range $3964 \AA-4567 \AA$ (LR2, $R=6400$ ) and observed spectra for a total of 101 blue hook and canonical blue HB/EHB star candidates and 17 empty positions for sky background.

For our analysis we used the pipeline-reduced data. For each exposure we subtracted the median of the spectra from the sky fibres from the extracted spectra. We corrected all spectra for barycentric motion. The individual spectra of each target star were cross-correlated with appropriate template spectra, in order to search for radial velocity variations. Since the few spectra per object did not permit a sophisticated period search, we determined the standard deviation of the radial velocity measurements for each star and compared it with the $\mathrm{S} / \mathrm{N}$ ratio of the spectra. As expected, the standard deviation of the radial velocity measurements decreases with increasing $\mathrm{S} / \mathrm{N}$ ratio. None of our target stars deviates significantly from this correlation, which would be the case for close binaries. Therefore none of our target stars appears to be in a close binary system. After verifying that there were no radial velocity variations, we co-added all spectra for each star. The co-added and velocity-corrected spectra were fitted with various model atmospheres: metal-free heliumrich non-LTE (Werner \& Dreizler 1999), metal-free helium-poor non-LTE (Napiwotzki 1997), and metal-rich helium-poor LTE (Moehler et al. 2000) as described in Moehler et al. (2004). This procedure yielded the effective temperatures, surface gravities, and helium abundances shown in Figs. 1 and 2. In this paper we concentrate only on the hottest HB stars with $T_{\text {eff }}>20000 \mathrm{~K}$.

\section{Results and discussion}

The helium-poor stars in Fig. 1 agree with the predictions of canonical evolutionary theory in that they populate the HB up to its hot end and then also contribute some evolved stars at higher effective temperatures and lower surface gravities. As we move to hotter stars ( $T_{\text {eff }} \gtrsim 30000 \mathrm{~K}$ ), we find a clump of stars populating the range in effective temperature and surface gravity between a fully mixed late hot flasher and the hot edge of the canonical HB. These stars show roughly solar helium abundance (cf. Fig. 2). The hottest stars lying along the evolutionary track of a fully mixed late hot flasher show the highest helium abundances, albeit with some hydrogen still in their atmospheres. In the temperature range $30000 \mathrm{~K}$ to $50000 \mathrm{~K}$ we find that $35 \%$ (15) of our stars are helium-poor $\left(\log \frac{n_{\mathrm{He}}}{n_{\mathrm{H}}}<-2\right), 51 \%$ (22) have solar helium abundance within a factor of $3\left(-1.5 \lesssim \log \frac{n_{\mathrm{He}}}{n_{\mathrm{H}}} \lesssim\right.$ $-0.5)$ and $14 \%(6)$ are helium-rich $\left(\log \frac{n_{\mathrm{He}}}{n_{\mathrm{H}}}>-0.4\right)$.

The helium-rich stars also show evidence for carbon enrichment as shown in Fig. 3, unlike the hot $\left(T_{\text {eff }}>30000 \mathrm{~K}\right)$ heliumpoor stars where no C II and C III lines were detected despite the higher $\mathrm{S} / \mathrm{N}$ in their spectra. We have constructed additional TLUSTY NLTE line-blanketed model atmospheres (Hubeny \& Lanz 1995; Lanz \& Hubeny 2003, 2007) for the atmospheric parameters of the helium-rich stars, assuming either scaled-solar 


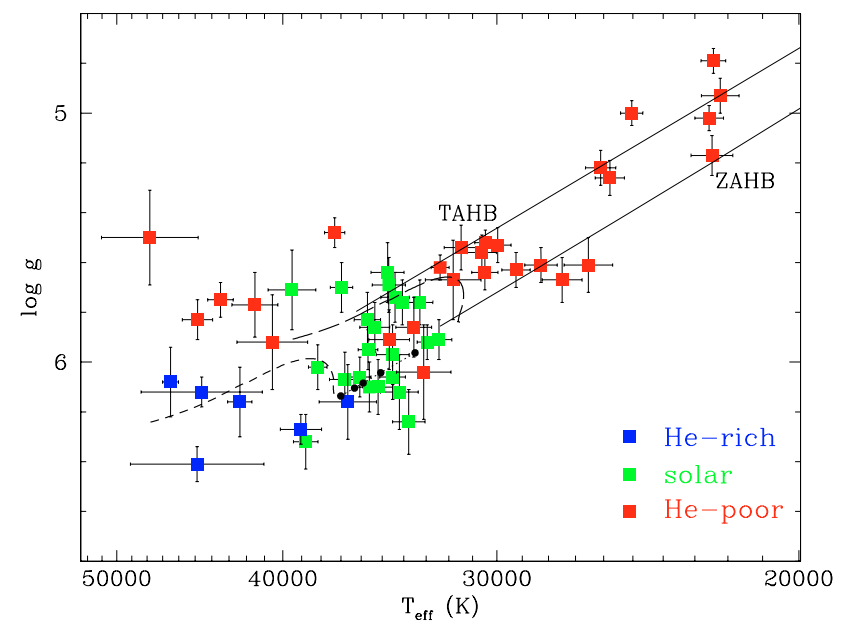

Fig. 1. The effective temperatures and surface gravities derived for our hottest target stars (formal errors only). Helium-poor, solar helium, and helium-rich stars are marked by red, green, and blue squares, respectively. The solid lines mark the canonical HB locus for $[\mathrm{M} / \mathrm{H}]=-1.5$ from Moehler et al. (2003). The tracks for an early hot flasher (longdashed line) and a late hot flasher (short-dashed line) show the evolution of such stars from the zero-age HB (ZAHB) towards helium exhaustion in the core (terminal-age $\mathrm{HB}=\mathrm{TAHB}$ ). The dotted line connects the series of ZAHB models computed by adding a hydrogen-rich layer to the surface of the ZAHB model of the late hot flasher. The small dots mark - with decreasing temperature - hydrogen layer masses of $0,10^{-7}, 10^{-6}, 10^{-5}, 10^{-4} M_{\odot}$ (for details see Moehler et al. 2002).

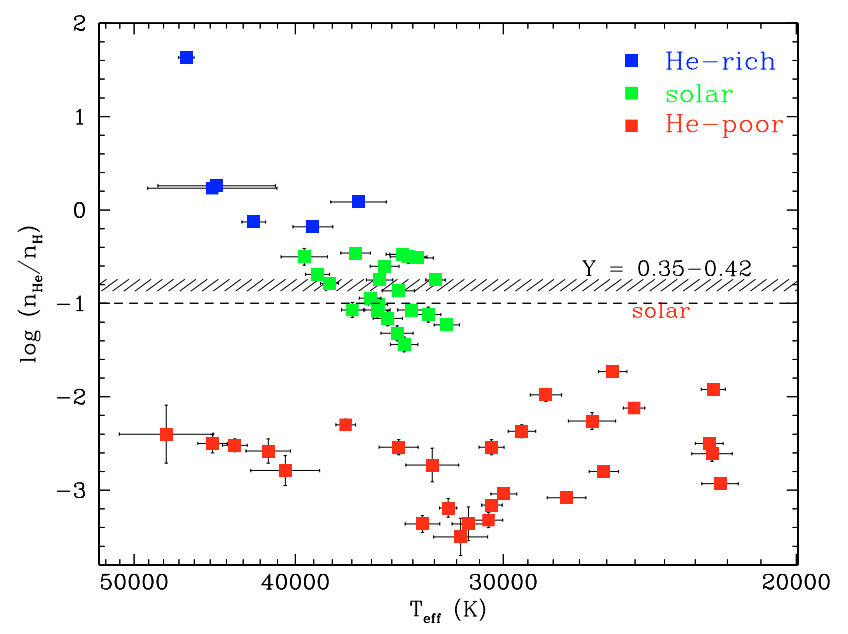

Fig. 2. The effective temperatures and helium abundances for our hottest target stars (formal errors only). The dashed line marks solar helium abundance, the hashed area marks the range for the helium-enrichment scenario. The symbols have the same meaning as in Fig. 1.

abundances appropriate for the dominant $\omega$ Cen metallicity $([\mathrm{M} / \mathrm{H}]=-1.5)$ or the carbon- and nitrogen-rich abundances predicted by the flash mixing scenario (mass fractions of $3 \%$ and $1 \%$, respectively). The comparison between observed and predicted C II and C III lines indicates that the helium-rich stars have a photospheric $\mathrm{C}$ mass fraction of at least $1 \%$ and up to $2-3 \%$ for the stars with the strongest lines. The typical line detection limit provides an upper limit of about $1 \%$ by mass for the $\mathrm{N}$ abundance. These $\mathrm{C}$ abundances represent a significant enhancement relative to the expected $\mathrm{C}$ abundance in $\omega$ Cen stars $(\lessgtr 0.1 \%$ at most for the most metal-rich stars).

Any discussion of the surface abundances in hot HB stars must consider the effects of diffusion. Fortunately the diffusion

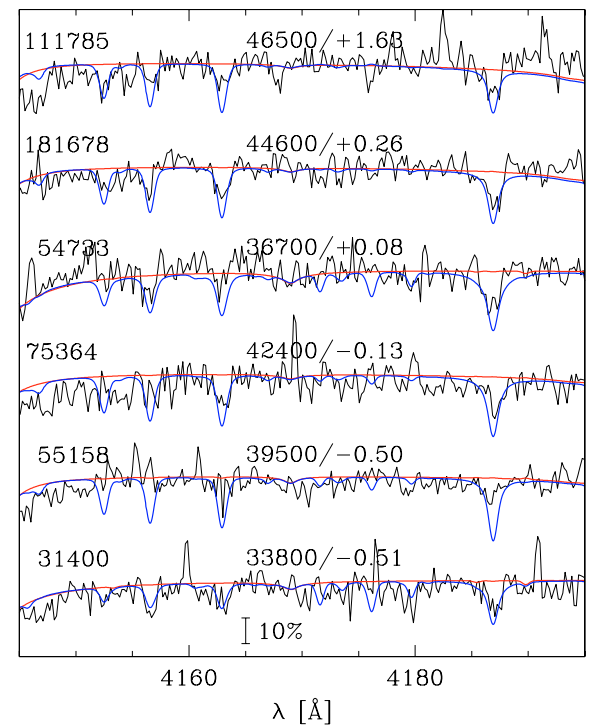

Fig. 3. Sample spectra of stars with super-solar helium abundance, compared to model spectra with the cluster carbon abundance for metalpoor stars (red) and a carbon abundance of $3 \%$ by mass (blue). The labels give the number of the star, its effective temperature, and its helium abundance $\log \frac{n_{\mathrm{He}}}{n_{\mathrm{H}}}$.

of $\mathrm{H}, \mathrm{He}$ and the $\mathrm{CNO}$ elements in the envelopes of stars following deep flash mixing has been investigated by Unglaub (2005). Not surprisingly, the results depend on the assumed mass loss rate and on the residual hydrogen abundance remaining after the flash mixing. For the low residual hydrogen abundance $X=$ 0.0004 predicted by the Cassisi et al. (2003) models, Unglaub (2005) found that a star will remain helium-rich with $\log \frac{n_{\mathrm{He}}}{n_{\mathrm{H}}} \approx$ $0 \ldots 2$ during most of the HB phase, in rough agreement with the helium-rich stars in Fig. 2. However, the residual hydrogen abundance following flash mixing is quite uncertain, since it depends on the mixing efficiency (Cassisi et al. 2003) and possibly on where the helium flash occurs along the white dwarf cooling curve. For a larger, but still low, residual hydrogen abundance of $X=0.004$, Unglaub (2005) found that diffusion can produce either a star with near solar helium abundance or a star that is helium-poor by the end of the HB phase. The stars with roughly solar helium abundances in Fig. 2 might be a consequence of such diffusion. The ZAHB models with a hydrogen-rich layer in Fig. 1 show that the effective temperature will decrease as the amount of surface hydrogen increases in qualitative agreement with the trend towards lower effective temperatures between the helium-rich and solar helium abundance stars in Fig. 2. Unglaub (2005) also noted that the diffusion efficiency increases substantially once the helium abundance approaches the solar value, leading to a rapid decrease in $\log \frac{n_{\mathrm{He}}}{n_{\mathrm{H}}}$ and perhaps accounting for the gap between the solar helium and helium-poor stars in Fig. 2. Diffusion in flash-mixed stars also leads to a decrease in the carbon and nitrogen abundances, which becomes more pronounced when the atmosphere is hydrogen-rich. Thus the carbon abundances derived here for the helium-rich stars may underestimate the initial carbon abundances in these stars.

The referee asked us to discuss the possibility that diffusion may not be active in all stars above $30000 \mathrm{~K}$. We consider the extreme case in which only the helium-poor stars in this temperature range are affected by diffusion. In this case the most helium-rich stars could still be reconciled with the late hot flasher scenario, but the same does not apply to the solarhelium stars. For the latter to be considered as the progeny of 
the helium-enriched main sequence stars, however, one would expect in Fig. 2 to see stars highly concentrated at $\log \frac{n_{\mathrm{He}}}{n_{\mathrm{H}}}=$ $-0.82 \ldots-0.74$ (i.e. $Y=0.38 \ldots 0.42$, Lee et al. 2005). Instead we see a rather large scatter, especially towards lower helium abundances. In addition, the progeny of the helium-enriched main sequence should not show strong carbon enhancements.

A puzzling effect becomes evident if one plots the spatial distribution of our target stars: Dividing the sample along a line running at $55^{\circ}$ counter-clockwise from east-west, the heliumpoor stars are evenly distributed (28:30 for all, 8:7 for those above $30000 \mathrm{~K}$ ), while the stars with roughly solar or supersolar helium abundance show a noticeable preference for the north-west section of the globular cluster (17:5 and 5:1, respectively). This peculiar spatial distribution appears similar to the reddening distribution observed by Calamida et al. (2005), who found a clumpy extinction variation with less reddened HB stars concentrated on the east side of the cluster (see their Fig. 5).

\section{Conclusions}

All of these results taken together offer strong support for the late hot flasher scenario as the explanation for the blue hook stars while posing a significant problem for the helium-enrichment scenario. This scenario predicts helium enrichment of up to $Y=$ $0.35 \ldots 0.42$, i.e. $\log \frac{n_{\mathrm{He}}}{n_{\mathrm{H}}}=-0.87 \ldots-0.74$. However, $40-30 \%$ of the stars above $30000 \mathrm{~K}$ show helium abundances in excess of these values, respectively. This result together with the observed carbon enhancement does not rule out the helium enhancement scenario, but it implies that additional processes are required to produce the hottest HB stars in $\omega$ Cen.

Acknowledgements. We thank the staff at the Paranal observatory and at ESO Garching for their excellent work, which made this paper possible. We also acknowledge the request from the ESO OPC to look into our already available data before applying again. Without their request these results would not have been found so soon. We thank the anonymous referee for his/her suggestions.

\section{References}

Bedin, L. R., Piotto, G., Anderson, J., et al. 2004, ApJ, 605, L125

Brown, T. M., Sweigart, A. V., Lanz, T., Landsman, W. B., \& Hubeny, I. 2001, ApJ, 562, 368

Cassisi, S., Schlattl, H., Salaris, M., \& Weiss, A. 2003, ApJ, 582, L43

Calamida, A., Stetson, P. B., Bono, G., et al. 2005, ApJ, 634, L69

Castellani, M., \& Castellani, V. 1993, ApJ, 407, 649

Castellani, V., Calamida, A., Bono, G., et al. 2007, ApJ, 663, 1021

D’Antona, F., \& Ventura, P. 2007, MNRAS, 379, 1431

D’Antona, F., Bellazzini, M., Fusi Pecci, F., et al. 2005, ApJ, 631, 868

D'Cruz, N. L., Dorman, B., \& Rood, R. T. 1996, ApJ, 466, 359

D’Cruz, N. L., O'Connell, R. W., Rood, R. T., et al. 2000, ApJ, 530, 352

Gratton, R. G., Sneden, C., Carretta, E., \& Bragaglia, A. 2000, A\&A, 354, 169

Hubeny, I., \& Lanz, T. 1995, ApJ, 439, 875

Kraft, R. P. 1994, PASP, 106, 553

Lanz, T., \& Hubeny, I. 2003, ApJS, 146, 417

Lanz, T., \& Hubeny, I. 2007, ApJS, 169, 83

Lanz, T., Brown, T. M., Sweigart, A. V., Hubeny, I., \& Landsman, W. B. 2004, ApJ, 602, 342

Lee, Y.-W., Joo, S.-J., Han, S.-I., et al. 2005, ApJ, 621, L57

Moehler, S., Sweigart, A. V., Landsman, W. B., \& Heber, U. 2000, A\&A, 360, 120

Moehler, S., Sweigart, A. V., Landsman, W. B., \& Dreizler, S. 2002, A\&A, 395, 37

Moehler S., Landsman W. B., Sweigart A. V., \& Grundahl, F. 2003, A\&A, 405, 135

Moehler, S., Sweigart, A. V., Landsman, W. B., Hammer, N. J., \& Dreizler, S. 2004, A\&A, 415, 313

Napiwotzki, R. 1997, A\&A, 322, 256

Newsham, G., \& Terndrup, D. M. 2007, ApJ, 664, 332

Norris, J. E. 2004, ApJ, 612, L25

Origlia, L., Ferraro, F. R., Bellazzini, M., \& Pancino, E. 2003, ApJ, 591, 916

Piotto, G., Villanova, S., Bedin, L. G., et al. 2005, ApJ, 621, 777

Piotto, G., Bedin, L. R., Anderson, J., et al. 2007, ApJ, 661, L53

Sweigart, A. V. 1997, The Third Conference on Faint Blue Stars, ed. A. G. D. Philip, J. Liebert \& R. A. Saffer (Schenectady: L. Davis Press), 3

Unglaub, K. 2005, The 14th European Workshop on White Dwarfs, ed. D. Koester, \& S. Moehler (San Francisco: ASP), ASP Conf. Ser., 334, 297

Werner, K., \& Dreizler, S. 1999, The Journal of Computational and Applied Mathematics, ed. H. Riffert, \& K. Werner (Amsterdam: Elsevier Press), 109, 65

Whitney, J. H., Rood, R. T., O'Connell, R. W., et al. 1998, ApJ, 495, 284

Zacharias, N., Urban, S. E., Zacharias, M. I., et al. 2004, AJ, 127, 3043 